\title{
2. Schlachtfeld der Repräsentation - Von der Pariser Kommune zum Nationalsozialismus
}

\begin{abstract}
Der erste fotografisch dokumentierte Krieg war der Krimkrieg (1853-1856), während es sich bei der ersten fotografisch festgehaltenen Revolution um die Pariser Kommune (18. März28. Mai 1871) handelt. Die Pariser Kommune begann als Aufstand der Nationalgarde nach der Kapitulation Frankreichs im Französisch-Preußischen Krieg und brach vor allem als Reaktion auf die mögliche Installation einer Monarchie aus. Es war die erste Revolution in der Geschichte, die breitere Arbeitermassen versammelte und unter anderem eine Säkularisierung und den Wegfall kirchlichen Eigentums, einen Schuldenerlass für die Arbeiter, die Abschaffung der Nachtarbeit, ein Verbot der finanziellen Bestrafung von Arbeitern, Arbeiterselbstverwaltung und soziale Demokratie sowie letztlich eine gänzlich neue territoriale Organisation Frankreichs zu erreichen beabsichtigte. In der letzten blutigen Maiwoche der Pariser Kommune kamen zwischen 10.000, manchen Schätzungen zufolge sogar 20.000 Kommunarden ums Leben, viele wurden bei Massenerschießungen und Vergeltungsaktionen ermordet. Eine Schlüsselrolle bei ihrer Ermordung sollte gerade die Fotografie spielen, da es der Regierung und der Armee anhand der Aufnahmen gelang, zahlreiche Kommunarden zu identifizieren. Als Medium zur Repräsentation des revolutionären Kampfes sowie später als grundlegende Identifikationstechnik in polizeilichen Archiven wurde die Fotografie daher zum neuralgischen Punkt des Aufstands. Sich des repressiven Potenzials der Fotografie nicht bewusst, ließen sich die Aufständischen während der Pariser Kommune gerne auf den Barrikaden ablichten, manchmal wurden sie sogar für die Fotografien mobilisiert.
\end{abstract}

„Mit erhobenem Stock als Signal für die Truppen (oder um dem Fotografen ein Zeichen zu geben) steht er vor dem leeren Weiß, gebannt, beleuchtet. Ein trotziger Aufständischer? Ein verspielter Poseur? Ein Engel, der bereit ist, gen Himmel zu fliegen? Ich schaue auf diesen Mann und frage mich, was er wohl zu sehen erwartete, als er vor der Kamera stand. Als sich die Blende schloss, hat er da die Macht der Geschichte gefühlt, die wie ein Sturm gegen seine Engelsflügel drängte? «1

Viele von ihnen stellten sich zum ersten Mal vor einen Fotografen. Naiv an die Unsterblichkeit glaubend, die die Fotografie versprach, wollten sie auf Papier gebannt werden. Die Fotografie war nämlich, wie Roland Barthes bemerkte, im zweiten französischen Kaiserreich 


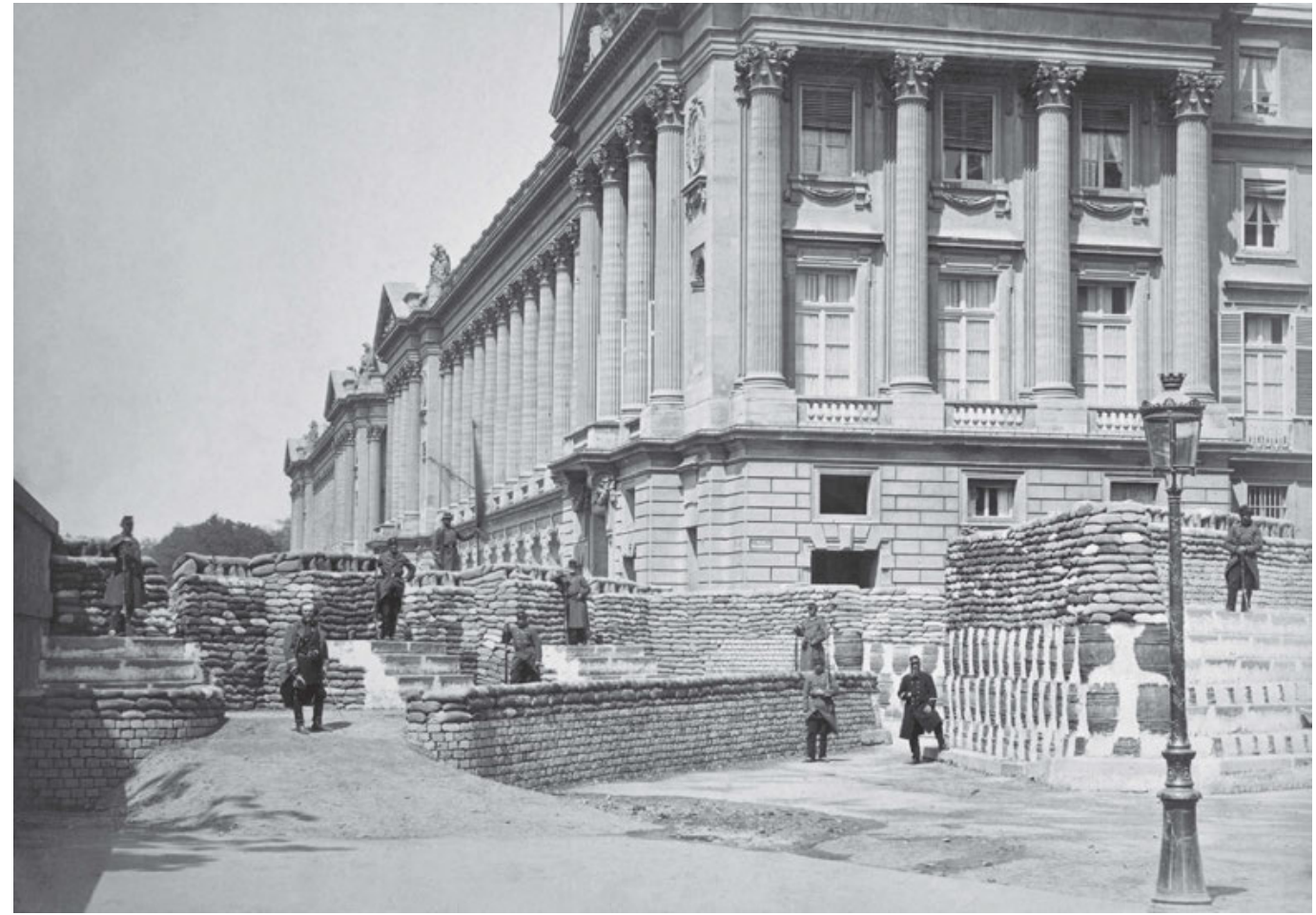

Barrikade zur Zeit der Pariser Kommune, in der Nähe des Ministeriums für Schifffahrt und des Hotels Crillon, 1871. Foto: Auguste Hippolyte Collard. New York, The Metropolitan Museum of Art - The Elisha Whittelsey Collection I 270204.

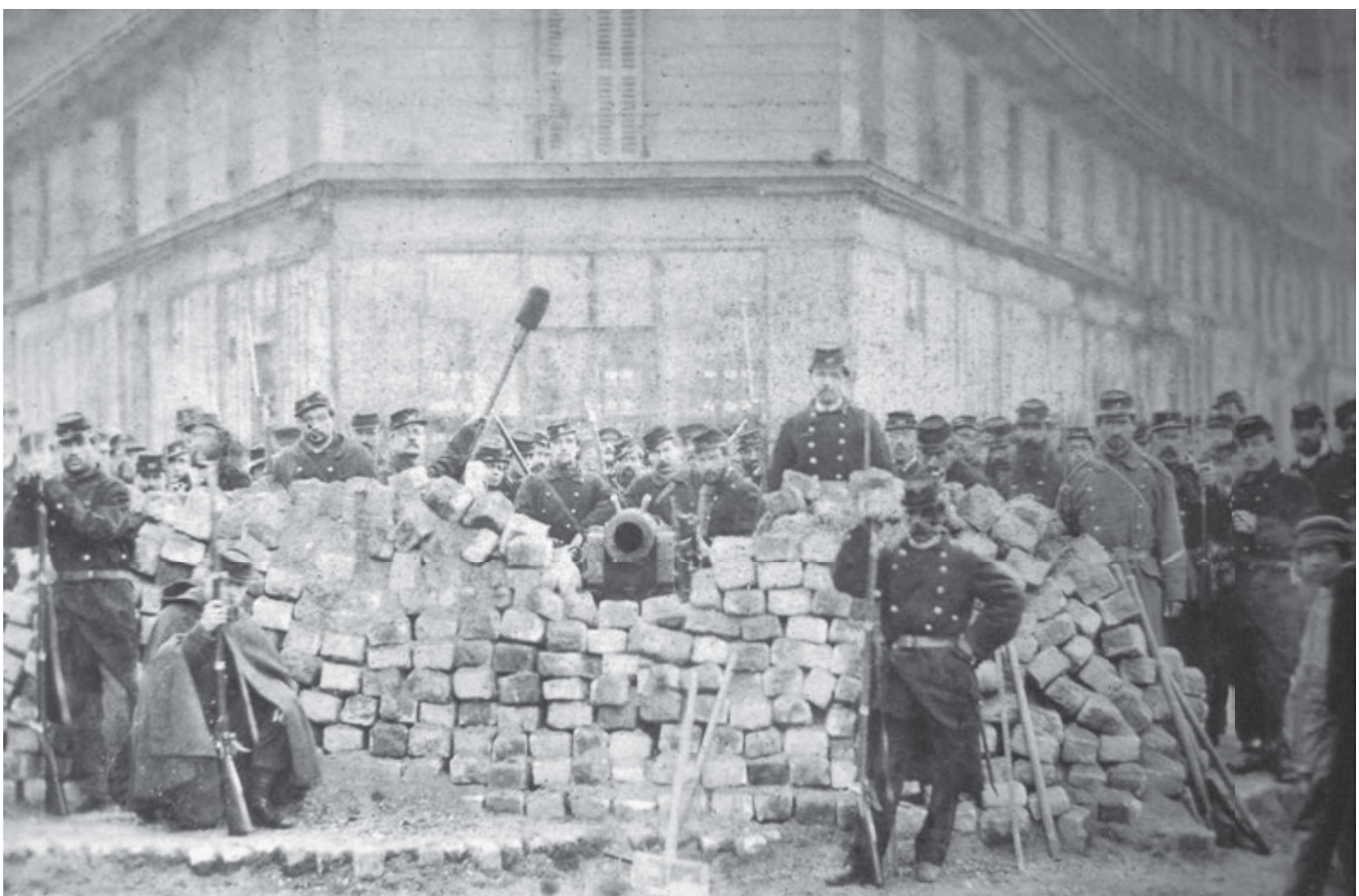

Barrikade Ecke Boulevard Voltaire und Boulevard Richard-Lenoir zur Zeit der Pariser Kommune, 1871.

Foto: Bruno Braquehais. Paris, Bibliothèque historique de la Ville de Paris. 
eng mit öffentlicher Identität, einem bürgerlichen Status und gesellschaftlicher Zugehörigkeit verbunden, die sich zumeist in der Bourgeoisie ${ }^{2}$ widerspiegelte und damals für einen kurzen Augenblick den niedrigeren Schichten zugänglich geworden war. In dieser Sehnsucht nach Unsterblichkeit liegt vielleicht auch die Tragödie der ganzen Geschichte verborgen. Durch ihr Streben nach Ewigkeit verloren sie den Kampf auf der Ebene der Repräsentation.

Zu den aktivsten Fotografen der Pariser Kommune zählte Bruno Braquehais. Mit Hilfe seiner Fotografien konnten die meisten Kommunarden nachträglich identifiziert werden. Der zweite und heute sicherlich bekanntere Pariser Fotograf, Eugène Appert, war zwar nicht vor Ort auf den Straßen aktiv, doch er ersann im Vergleich zu seinen Konkurrenten eine perfidere Form, um zu Geld zu kommen. Er fotografierte mit dem Segen der Regierung für deren, aber auch für seinen eigenen Bedarf die verhafteten und eingesperrten Kommunarden, während er im Gegenzug uneingeschränkte Urheberrechte für die weitere Verwendung der Bilder erhielt. Da die Nachfrage des Publikums nach diesen Fotografien stetig stieg, beschloss Appert einzelne Ereignisse zu rekonstruieren und engagierte zu diesem Zweck sogar professionelle Schauspieler. Zum ersten Mal in der Geschichte entstand ein fotografisches Tableau vivant, und zwar für die Bedürfnisse der Regierung sowie zwecks Profitsteigerung des Fotografen. Appert schuf die ersten nachgestellten Bilder in der Geschichte der Fotografie, beteiligte sich dadurch an der Propaganda gegen die Kommune und trug zur Repression gegen die verhafteten Kommunarden bei. ${ }^{3}$ Auf den Originalen haben alle in den Kerker geworfenen Kommunarden nach der Niederlage einen müden und erschöpften Gesichtsausdruck, ihre Kleidung ist verdreckt und ihre Erniedrigung mehr als offensichtlich. Der einstige Kriegsminister Oberst Louis Rossel, der sich gleich zu Beginn des Aufstands den Kommunarden angeschlossen hatte und später als eine Schlüsselfigur der Pariser Kommune galt, wurde im September 1871, zwei Monate vor seiner Erschießung, im Gefängnis von Appert porträtiert. Nachträglich montierte dieser auf Rossels Porträt den Körper eines anderen Mannes, den er in seinem Fotostudio fotografiert hatte. ${ }^{4}$ Dabei ging Appert so weit, dass er sogar die Aufnahme von Rossels Erschießung nachstellte. Nicht wegen seiner fotografischen und künstlerischen Bedeutung ging der jeglichen ethischen und moralischen Normen ferne Eugène Appert in die Geschichte der Fotografie ein, sondern vielmehr aufgrund der Tatsache, dass er als Urheber der fotografischen Manipulation gilt, die wegen der Annahme der Authentizität der Fotografie das naivere Publikum geschickt zu täuschen vermochte. Dies war der Beginn der Manipulation mit Hilfe von Fotografien.

\footnotetext{
»Apperts Kompositporträts erscheinen mir umso heimtückischer, nicht nur weil es sich um gefälschte Bilder handelt, die vorgeben, real zu sein, sondern auch weil sie möglicherweise alle fotografischen Porträts als Fiktion enttarnen, indem sie sogar das fragile Decorum der gefangenen Kommunarden als wenig mehr denn Verkleidung enttarnen. $\ll^{5}$
}

\footnotetext{
2 Ebd., S. 63.

3 Field, Peter. History in the Making. In: Journal, Nr. 18, 2010. Siehe http://worker01.e-flux.com/pdf/article_161.pdf. [Abruf am 22.01.2021].

4 Przyblyski, Jeannene M. Revolution at a Standstill: Photography and the Paris Commune of 1871. In: Yale French Studies, Nr. 101, 2001, S. 67.

5 Ebd., S. 77.
} 


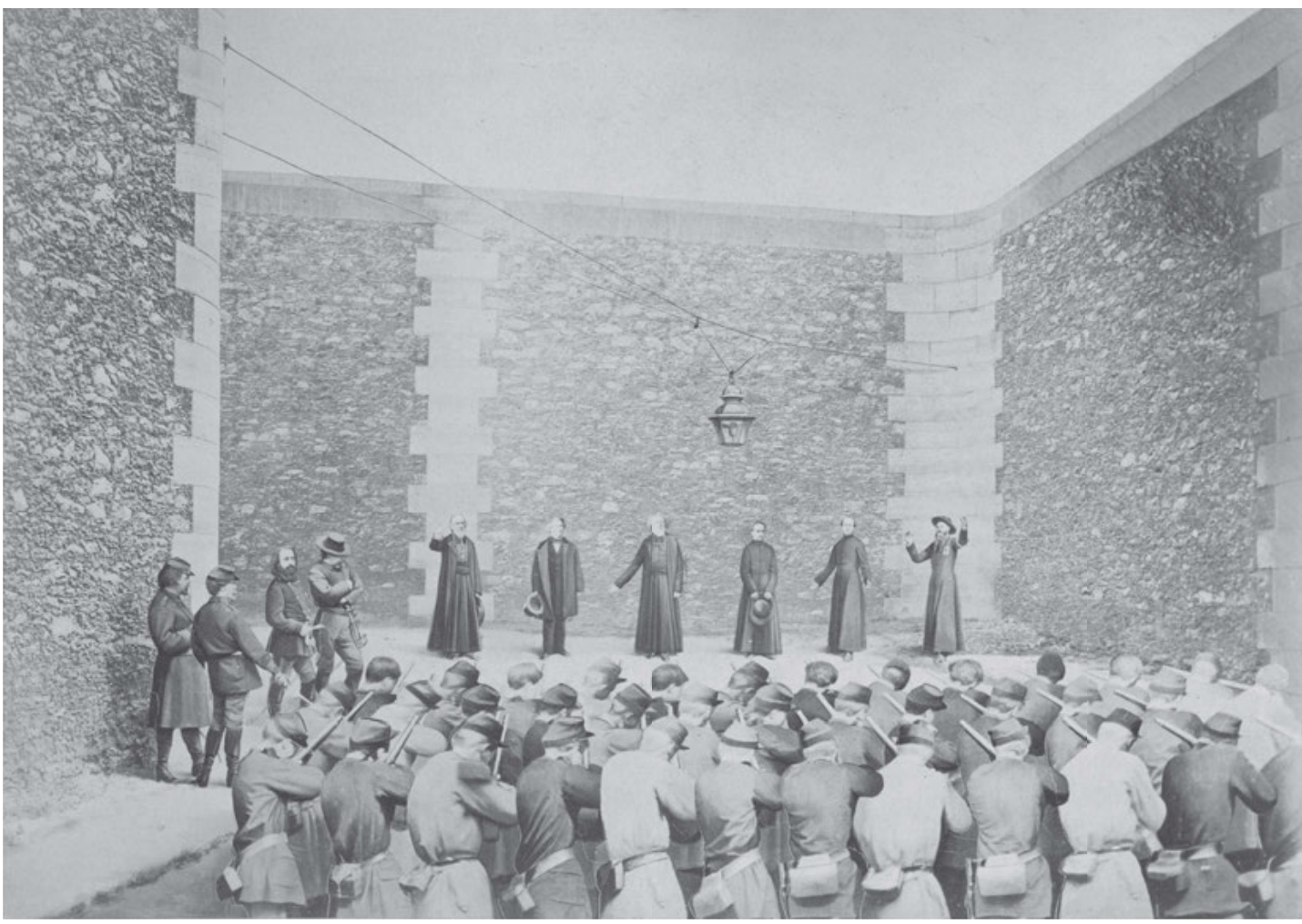

Geiselerschießung, Gefängnis De La Roquette, 24. Mai 1871. Fotograf unbekannt. New York, The Metropolitan Museum of Art | 302335, Joyce F. Menschel Photography Library Fund, 2012.

Obwohl die Erfindung der Carte de Visite im Jahr 1854 die Fotografie für eine größere Personenzahl erschwinglich machte, war sie dennoch für die Arbeiterklasse und die ärmste Stadtbevölkerung unerreichbar. Kultur und Kunst blieben in erster Linie der Bourgeoisie vorbehalten, die im Unterschied zur Arbeiterklasse Zeit für Müßiggang hatte. Der Erfinder der »fotografischen Visitenkarten«, Eugène Disdéri, hielt ebenfalls die Kommunarden auf den Barrikaden, aber auch ihre Leichen fest. Seine Fotografien zeigten für Versailles Aufständische, die ihre gerechte Strafe erhielten, während sie für die Kommunarden Beleg für die unwahrscheinlich brutale Vergeltung und Trophäenfotografien der begangenen Verbrechen waren.

Zur Zeit der Pariser Kommune wurden Fotografien der Aufständischen in allen Zeitungen veröffentlicht, was der Polizei und der Armee bei der Identifizierung der Revolutionäre half, nachdem das damals im Hôtel de Ville untergebrachte polizeiliche Archiv niedergebrannt worden war. Der erneuten Einrichtung eines polizeilichen Archivs widmete sich der Pariser Inspektor Alphonse Bertillon. Bis 1883 entwickelte er darüber hinaus für die Installation dieses Archivs die anthropometrische Ordnung und Statistik. Bertillons Archiv bildete dann auch die Grundlage für die Einrichtung moderner Polizeiarchive, wobei die Art des Fotografierens von Verhafteten, nämlich frontal mit dem Gesicht im Vollbild - die sogenannten Mugshots - bis zum heutigen Tag polizeilicher Standard geblieben ist. Dies wirkte sich ebenfalls auf die Entwicklung moderner biometrischer Identifikations- und Überwachungsmethoden aus. 
Bertillons Arbeit bildete die Voraussetzung dafür, dass sein britischer Zeitgenosse Francis Galton, Erfinder des fotografischen Kompositporträts und des synekdochischen Fingerabdrucks, ein Archiv einrichten konnte, das auf biologischem Determinismus und Eugenik basierte. Demzufolge könne mit Hilfe der Fotografie die Zahl derjenigen gemindert werden, die zu Armut und Elend verdammt sind. Auch den Anstieg der urbanen ärmlichen Bevölkerung glaubte man stoppen zu können, indem man sich auf die rassistischen Theorien von der Superiorität der weißen Rasse gegenüber den Schwarzen aus Afrika, aber auch gegenüber dem Proletariat berief. ${ }^{6}$ Galtons Kompositporträts ebneten den Weg für die Entstehung der essenzialistischen Rassenanthropologie sowie für das Konstruieren von Porträts sogenannter »minderwertiger« Rassen und Nationen.

Mit dem Aufkommen des Nationalsozialismus kam es zur Verschmelzung von Fotografie und Eugenik im Dienste der Rassentheorie. Aus diesem Grund wurden die Fotografien von August Sander vernichtet, der beim Porträtieren deutscher Bürger für sein Buch Antlitz der Zeit Hunderte von Aufnahmen anfertigte, die nicht dem arischen Paradigma entsprachen.

Nach der Machtergreifung durch die Nationalsozialisten im Jahr 1933 entwickelte sich die Fotografie zur mächtigen und einflussreichen Propagandawaffe, aber auch zur Technik, die der Etablierung neuer, vor allem antisemitischer Theorien diente. Im Naturhistorischen Museum in Wien wurde im Mai 1939 eine Ausstellung mit dem Titel Das körperliche und seelische Erscheinungsbild der Juden organisiert, die Dr. Josef Wastl, Leiter der Abteilung für Anthropologie im selben Museum und seit 1932 Mitglied der NSDAP, eröffnete. Während die bereits gezeigte Ausstellung Der ewige Jude avantgardistische Werke von jüdischen Künstlern als »entartete Kunst" präsentierte, rechtfertigte diese Ausstellung ihren pseudowissenschaftlichen Ansatz mit zahlreichen Fotografien von Juden aus polizeilichen Archiven, geleitet von der Absicht, das »verräterische" Antlitz der Juden zu zeigen.7 Im September 1939 ging Wastl noch einen Schritt weiter und führte Untersuchungen auf der Basis von insgesamt 440 Juden durch, die im Wiener Fußballstadion interniert waren. ${ }^{8}$ Über eine spätere Wiener Ausstellung zum »Kampfgebiet im Südosten«, an der auch Serbien unter der Führung von Milan Nedić teilnahm, schrieb die Zeitung Srpski narod (Serbisches Volk) am 1. Juli 1944, dass »Trophäen der kommunistischen Partisanenbanden« und Fotografien getöteter Partisanen sowie einige Partisanen abgebildet sind, deren Einheiten serbische Nationalisten liquidiert hätten. ${ }^{9}$

Bertillons Archiv öffnete der Eugenik die Tür, die ihrerseits die Basis für die nationalsozialistischen Rassentheorien darstellte. Die an der jugoslawischen Partisanenbewegung beteiligten Slawen fielen diesen Theorien zum Opfer, kämpften aber auch gegen sie an. Zudem bildete sich innerhalb der Partisanenbewegung eine besondere künstlerische und fotografische Geschichte heraus, wobei von Beginn des bewaffneten Widerstands an das

\footnotetext{
6 Konjikušić, Davor. Fotografija i moć. Zagreb: Akademie für Dramenkunst (Diplomarbeit) 2014, S. 23-24.

7 Renyi, Andras. Time to Gaze. In: Ders. (Hrsg.). Col Tempo. The W. Project, Peter Forgac's Installation. Budapest: Muscarnok Non-profit Ltd. 2009, S. 19.

8 Berner, Margit. The Nazi Period Collections of Physical Anthropology in the Museum of Natural History. Wien 2009. Siehe http:// coltempo.hu/catalog/margit_berner.html [Aufruf am 22.01.2021].

9 Milosavljević, Olivera. Potisnuta istina. Kolaboracija u Srbiji 1941-1944 (=Ogledi, Nr. 7). Belgrad: Helsinški odbor za ljudska prava u Srbiji 2006, S. 30.
} 
Bewusstsein ausgeprägt war, dass der Sieg ebenso auf der Ebene der Repräsentation errungen werden musste. Die Partisanen, die in den Freiheitskampf zogen, trugen siebzig Jahre später jene Fahne, unter der auch die Pariser Kommunarden gekämpft hatten. ${ }^{10}$ Seit der Pariser Kommune befinden sich soziale Bewegungen in einer schwächeren Position gegenüber der vorherrschenden Ordnung. Diese hat die Kontrolle über die Produktions- und Distributionskanäle der Propaganda inne, was die Kommunarden leider viel zu spät eingesehen und am Ende mit dem eigenen Leben bezahlt haben. Die Partisanen indes hatten verstanden, dass die Darstellung stets durch Repräsentation und Ideologie ${ }^{11}$ vermittelt wird und die Notwendigkeit bestand, den Kampf auch auf dem (Schlacht-)Feld der Repräsentation zu gewinnen. 